\title{
Estudo da Microestrutura Formada no Processo de Soldagem por Atrito em Aço C-Mn com Pino Consumível
}

\author{
Microstructural evaluation of a C-Mn steel welded by the Friction Hidro-Pillar Process
}

\author{
Cleber Rodrigo de Lima Lessa ${ }^{1}$, Marcelo Freitas Caregnato ${ }^{1}$, Pedro Henrique Costa Pereira da Cunha ${ }^{1}$, Mariane Chludzinski ${ }^{1}$, Telmo \\ Roberto Strohaecker', Márcio Levi Kramer de Macedo', Marcelo Torres Piza Paes ${ }^{2}$ \\ ${ }^{1}$ UFRGS, PPGEM/Lamef, Porto Alegre, RS, Brasil, mail: lima lessa@yahoo.com.br \\ ${ }^{2}$ Petrobras, Cenpes, Rio de Janeiro, RJ, Brasil
}

\begin{abstract}
Resumo
A soldagem por arco elétrico é a técnica mais empregada para reparar estruturas de grande porte, mas apresenta desvantagens associadas à solidificação do metal de solda. Então a utilização de processos de solda por atrito, especificamente o processo FHPP (Friction Hydro Pillar Processing) que consiste na utilização de pinos para reparo por atrito, apresenta vantagens por ser um processo realizado no estado sólido. Menores temperaturas estão envolvidas e não existe influência do ambiente externo, minimizando os problemas nos reparos estruturais. Este trabalho teve como objetivo observar a variação dos parâmetros do processo e os efeitos desses na microestrutura e qualidade do reparo. O estudo comparou diferentes forças axiais nos reparos com aço C-Mn através de metalografias e perfis de microdureza. Uma força axial maior resultou em um menor tempo de processo, em uma microestrutura mais homogênea e evitou os defeitos "falta de adesão" na zona de ligação e "cisalhamento a quente" do pino.
\end{abstract}

Palavras-chave: FHPP; Microestrutura; Força Axial; ZTMA (zona termo-mecanicamente afetada); ZTA (zona termicamente afetada).

\section{Abstract:}

The arc welding it is the most used technique to repair large structures, however, has disadvantages that result from the solidification of the weld metal. So the use of friction welding, specifically the FHPP (friction hydro pillar processing) process that consists on using plugs for friction repair, presents advantages because it is a process that is carried out on the solid state. Lower temperatures are involved and do not have the influence of the external environment, minimizing the problems on structure repairs. This work has the objective of observe the process parameters variation and their effects on microstructure and on the quality of the repair. The study compared different axial forces on repairs whit C-Mn steel through of metallographic and microhardness profiles. A higher axial force results in less time of process, in a more homogeneous microstructure, and avoided the defects "noncompliance" in the bounding zone and "hot shear" of the plug.

Key-words: FHPP; microstructures; Axial Force; TMAZ; HAZ.

\section{Introdução}

O processo FHPP (Friction Hidro Pillar Processing) tem como objetivo principal atender a demanda de reparos de estruturas, tais como plataformas e navios utilizados na indústria de óleo e gás [1-4]. Nos reparos, o processo de soldagem por atrito torna-se vantajoso devido ao baixo nível de calor gerado, ausência de fumos e gases residuais excessivos além da exposição da junção em ambientes agressivos onde não pode ser facilmente aplicada a devida proteção gasosa. Adicionalmente, pelo caráter de processo de soldagem no estado sólido, é possível obter juntas soldadas com a ausência de problemas associados ao desenvolvimento de trincas por ação do hidrogênio e ao processo de solidificação [5]. Outra vantagem do processo é que esse pode ser facilmente automatizado, garantindo reprodutibilidade e qualidade controladas da junta soldada [6]. Existem estudos

(Recebido em 02/09/2010; Texto final em 11/01/2011). recentes da aplicação do processo FHPP em ligas $\mathrm{Mg}-\mathrm{Al}$ para indústria automotiva devido a sua baixa densidade [8].

O processo conduzido inteiramente no estado sólido é importante, pois esse não atinge calor suficiente para fundir o material e assim limita a extensão das transformações metalúrgicas que podem fragilizar o material. Os fatos mencionados aumentam a confiabilidade do reparo e reduzem tempo e custo. O processo FHPP consiste na utilização de um pino consumível com rotação entre 1000 a $2000 \mathrm{rev} / \mathrm{min}$ e de uma força axial aplicada que faz com que esse pino entre em contato com fundo de furo cônico para criar o calor a partir do atrito e da deformação, que plastifica o pino contra o metal base e dessa forma preenche o furo (Figura 1) [6-8].

Os parâmetros de controle do processo são a velocidade de rotação, "burn off" (comprimento do pino consumível processado), força axial aplicada sobre a chapa e o tempo de aquecimento. A combinação desses fatores influencia diretamente na qualidade final da solda para que não ocorra fragilização microestrutural, falta de preenchimento do furo e 
a falta de adesão entre os dois corpos. Por esses motivos se faz necessário o estudo de diferentes cargas axiais aplicadas [9].
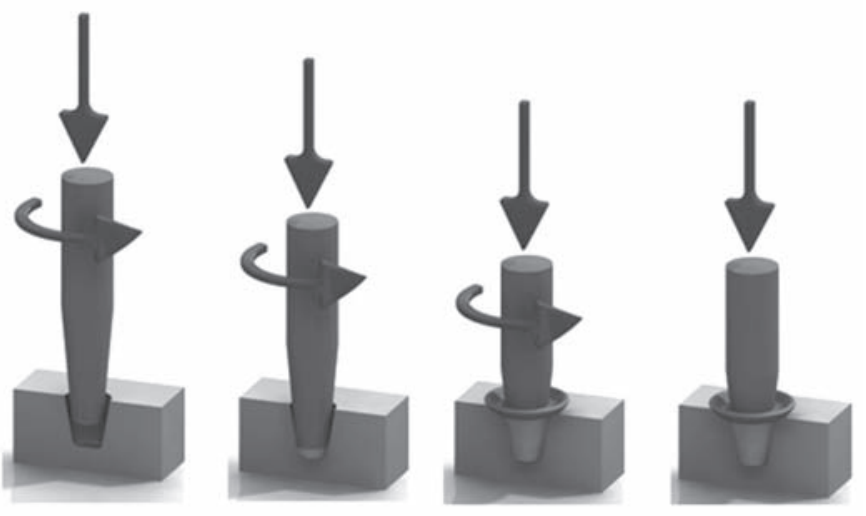

Figura 1 - Desenho esquemático do processo.

O objetivo do presente trabalho é estudar a microestrutura formada variando a força axial aplicada sobre o pino consumível de aço C-Mn. Utilizou-se equipamento estacionário de grande inércia, convencionalmente utilizado para soldagem axial de barras cilíndricas.

Diversos testes preliminares foram realizados no TWI Ltd da Inglaterra variando a velocidade rotacional e o ângulo do pino de forma a verificar a ocorrência do total preenchimento da cavidade e se a solda era aprovada no ensaio de dobramento [9]. A influência da força axial (ou pressão) na formação da microestrutura foi avaliada através de caracterização microestrutural e perfis de microdureza.

\section{Materiais e Métodos}

As soldas foram realizadas utilizando uma máquina modificada de soldagem por atrito NEI - John Thompson modelo FW-13 (pertencente ao centro de pesquisas TWI) com uma potência máxima de $43 \mathrm{~kW}$ e força axial limite de 480 $\mathrm{kN}$ e peso de 7 toneladas, o que confere resistência e rigidez suficientes para poder reagir a cargas aplicadas elevadas. Todas as amostras foram fabricadas utilizando uma velocidade rotacional de $1550 \mathrm{rev} / \mathrm{min}$.

Para os ensaios foram utilizadas as seguintes forças axiais: $50 \mathrm{kN}, 200 \mathrm{kN}$ e $400 \mathrm{kN}$. Os materiais utilizados nesse trabalho foram: I - Chapa - 200 x 140 x 30; material - Aço EN 10025 (Antigo BS4360 Gr50D); II Pinos consumíveis - $30 \mathrm{~mm}$ (diâmetro); material - Aço BS970-3 150M19 (Originalmente classificado como EN14, obtidos a partir de barras cilíndricas laminadas à quente). As composições químicas foram realizadas em um espectrômetro de emissão óptica da marca SPECTRO, modelo Spectrolab. Os resultados de composição química das chapas onde foram feitos os reparos por FHPP e dos pinos utilizados estão na Tabela 1.
Os perfis de microdureza e as análises metalográficas foram realizados nas diversas regiões da junta soldada. As medidas de microdureza foram realizadas na escala Vickers (HV) com carga de 300 grf em um microdurômetro da marca INSTRON modelo Tukon 2100 B. As análises metalográficas objetivaram a identificação das microestruturas (fases e constituintes) utilizando microscopia de luz (ML) e eletrônica de varredura (MEV). Os equipamentos utilizados foram: microscópio de luz OLYMPUS (modelo BX51M, com câmera acoplada, reflexão de luz branca, luz negra e luz polarizada simplificada e microscópio eletrônico de varredura SHIMADSU (modelo SSX-550 SUPERSCAN). As amostras foram preparadas segundo procedimento padrão de preparação metalográfica (baseado na Norma ASTM xx) e polidas com pasta de diamante. Após isso, as amostras analisadas foram atacadas quimicamente com as soluções: Nital $2 \%$ com o objetivo de revelar as regiões e microestruturas da junta soldada; Picral com o objetivo de relevar o tamanho de grão austenítico e Le Pera com o objetivo de revelar constituinte MA (constituinte contendo Martensita e Austenita). A geometria e dimensões da junta a ser soldada são apresentadas na Figura 2.

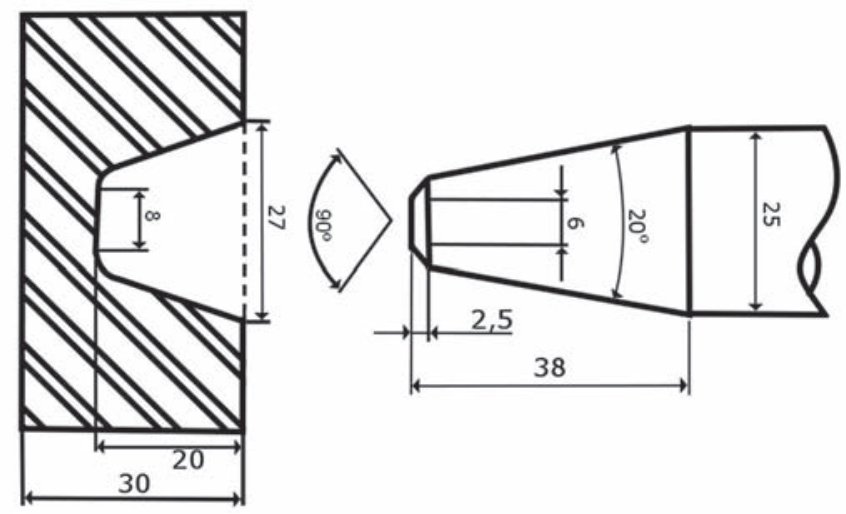

Figura 2 - Croqui: pino e junta.

\section{Resultados e Discussão}

\subsection{Análise Microestrutural pino e chapa}

Pela observação da microestrutura do pino e da chapa, (Figura 3), foi constatada a ocorrência do fenômeno conhecido como bandeamento. O bandeamento é uma condição microestrutural, desenvolvida principalmente em aços ligados e de resfriamento lento, os quais apresentam bandas alternadas de ferrita e perlita, paralelas ao sentido de laminação/trefilação [10].

\subsection{Parâmetros e Macrografias}

A energia mecânica consumida para superar as forças de atrito é dissipada na forma de calor nas superfícies em

Tabela 1 - Composição química dos componentes (\% em massa).

\begin{tabular}{|c|c|c|c|c|c|c|c|c|c|c|c|c|c|c|c|}
\hline & $\mathrm{C}$ & Si & Mn & $\mathbf{P}$ & $\mathbf{S}$ & $\mathrm{Cr}$ & Mo & $\mathbf{N i}$ & Al & $\mathrm{Cu}$ & $\mathbf{T i}$ & $\mathbf{V}$ & Sn & B & $\mathrm{Fe}$ \\
\hline Chapa & 0,16 & 0,40 & 1,31 & 0,012 & 0,007 & 0,018 & $<0,005$ & 0,022 & 0,037 & 0,021 & 0,001 & $<0,001$ & $<0,001$ & $<0,0001$ & Balanço \\
\hline Pino & 0,19 & 0,29 & 1,42 & 0,017 & 0,040 & 0,200 & 0,022 & 0,124 & 0,005 & 0,27 & 0,013 & 0,005 & 0,018 & 0,0005 & Balanço \\
\hline
\end{tabular}



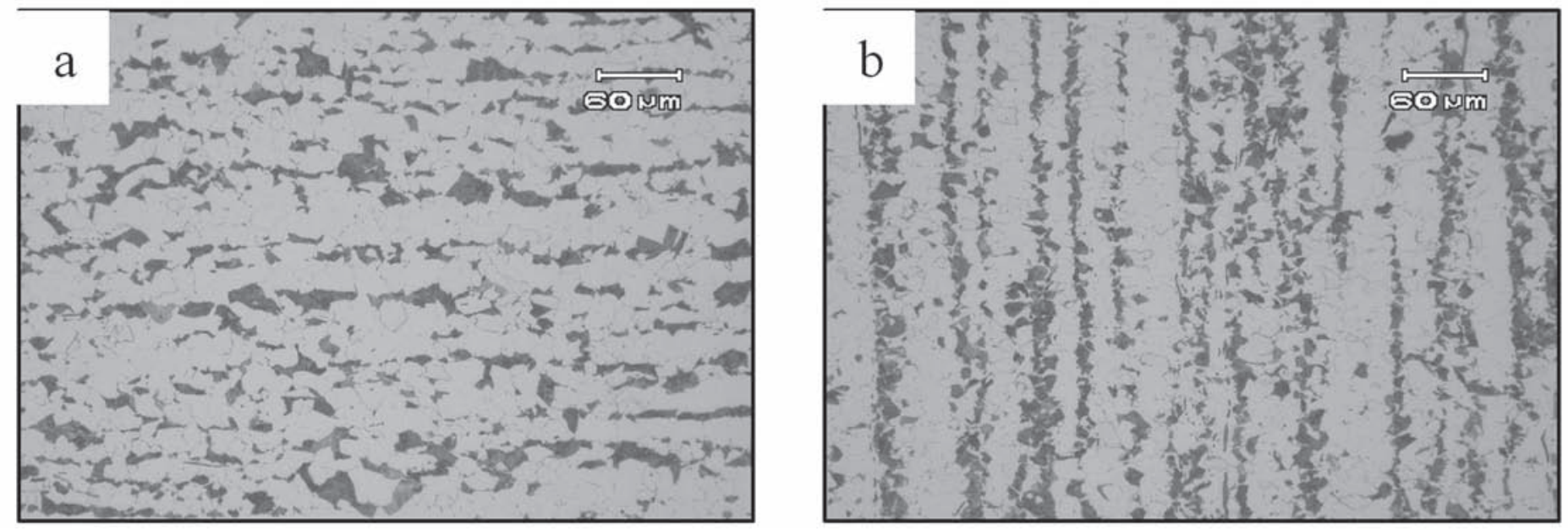

Figura 3 - (a) Microestrutura exclusiva da chapa, (b) Microestrutura exclusiva do pino.

contato, como também no corpo das projeções que são deformadas plasticamente. Na Tabela 2 estão listados o tempo de processamento e outros fatores do processo. Quanto maior o tempo em processo, mais energia está sendo despendida e, conseqüentemente, maior a quantidade de calor. Existe uma fonte ativa geradora de calor em uma fina camada do metal bem próxima da superfície de contato [11]. No presente trabalho a camada próxima à superfície de contato vai ser tratada como “zona de ligação (ZL)”. A Tabela 2 apresenta os parâmetros de soldagem utilizados.

Tabela 2 - Parâmetros de soldagem.

\begin{tabular}{|l|c|c|c|}
\hline Força axial $[\mathrm{kN}]$ & 50 & 200 & 400 \\
\hline Rotação [rev/min] & 1550 & 1550 & 1550 \\
\hline Burn off [mm] & 12 & 12 & 12 \\
\hline Tempo [s] & 31,3 & 5,2 & 2,0 \\
\hline
\end{tabular}

Segundo Ellis [12], o parâmetro força axial controla de forma preponderante os tempos de soldagem e também atua no perfil e nas características da ZTA. As macrografias (Figura 4) obtidas confirmam essa observação. A penetração de queima nas cargas de 50, 200 e $400 \mathrm{kN}$ foi de 20, 23 e $22 \mathrm{~mm}$, respectivamente. As maiores forças levaram a um estreitamento da zona termicamente afetada (ZTA) e da zona termo-mecanicamente afetada (ZTMA). Entende-se que a zona termo-mecanicamente afetada é a parte em que houve uma modificação perceptível da microestrutura por meio do calor e da força aplicada, principalmente na etapa do forjamento. O forjamento neste processo é a etapa após o aquecimento que consiste em manter por um tempo adicional a força axial entre as duas partes que estão sendo soldadas. A carga utilizada no forjamento é igual ou maior do que a carga utilizada durante o estágio de aquecimento $[9,11]$.

Foram realizadas a quantificação e a classificação (Tabela 3) conforme Dubé [13] e Thewly [14], das fases presentes na ZTMA em relação às cargas. A penetração de queima da carga de $200 \mathrm{kN}$ foi maior que a de $400 \mathrm{kN}$ provavelmente pelo fato do tempo de processamento ser maior na de $200 \mathrm{kN}$, que gera mais calor e facilita o forjamento (Figura 4).
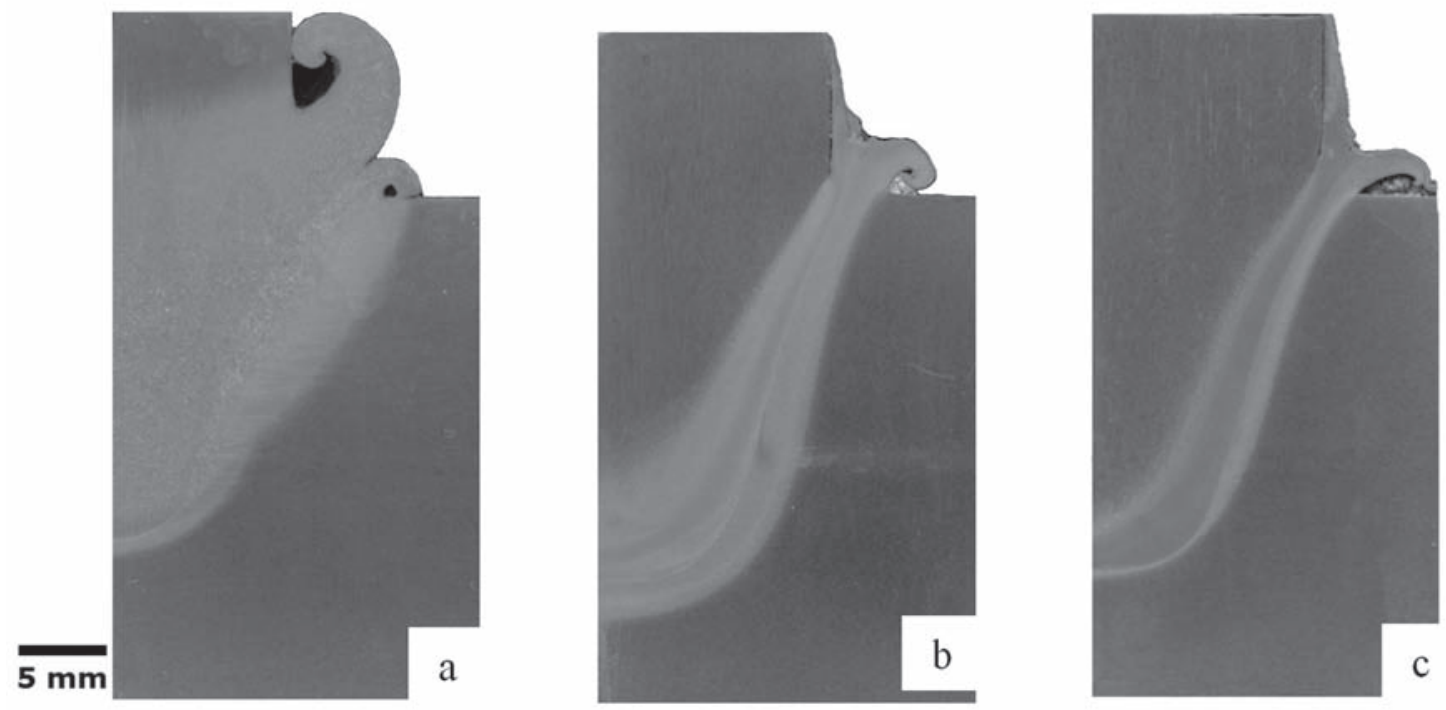

Figura 4 - Macrografias: (a) Carga de 50 kN, (b) Carga de 200 kN e (c) Carga de 400 kN. 
Tabela 3 - Quantificação das fases para as diferentes cargas.

\begin{tabular}{|l|l|c|c|}
\hline Carga & \multicolumn{1}{|c|}{ Microestrutura } & $\begin{array}{c}\text { \% } \\
\text { médio }\end{array}$ & $\begin{array}{c}\text { Desvio } \\
\text { padrão }\end{array}$ \\
\hline \multirow{3}{*}{$50 \mathrm{kN}$} & ferrita acicular [AF] & & \\
& bainita [B] & 2,13 & 0,18 \\
& agregado de ferrita e carboneto [FC] & 39,00 & 10,61 \\
& ferrita com fase alinhada/ & 13,30 & 1,41 \\
& Widmänsttaten [FS(A)] & 45,58 & 10,43 \\
& & & \\
\hline \multirow{3}{*}{$200 \mathrm{kN}$} & ferrita acicular & 5,75 & 0,35 \\
& bainita & 37,50 & 4,95 \\
& ferrita com segunda fase alinhada/ & 56,75 & 9,12 \\
& Widmänsttaten & & \\
\hline \multirow{2}{*}{$400 \mathrm{kN}$} & martensita & 91,00 & 2,51 \\
& ferrita primária & 9,00 & 3,43 \\
\hline
\end{tabular}

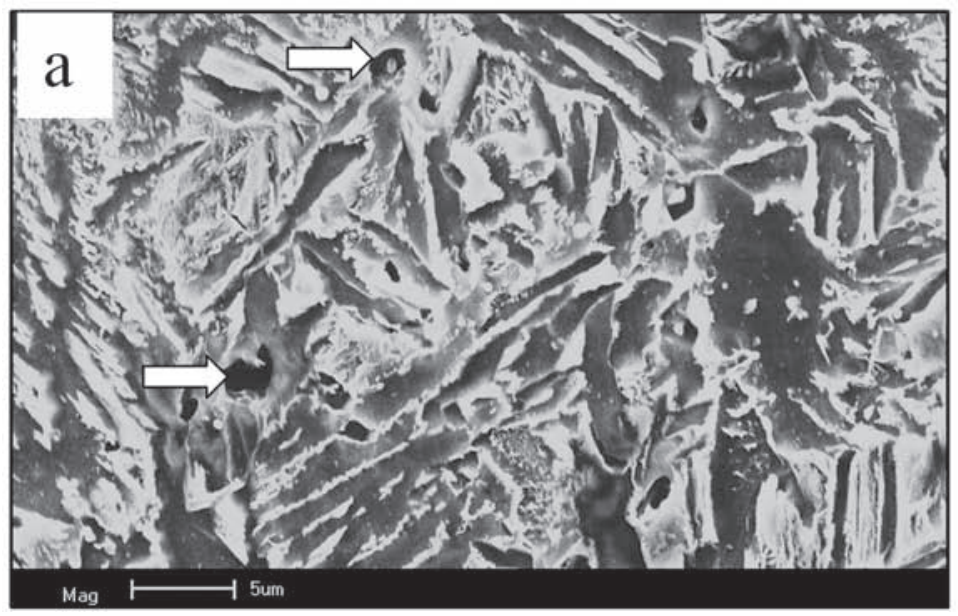

Figura 5 - Micrografias em MEV com o detalhe de falta de adesão na ZL da carga de 50 kN (MEV). Em (b) com maior aumento.
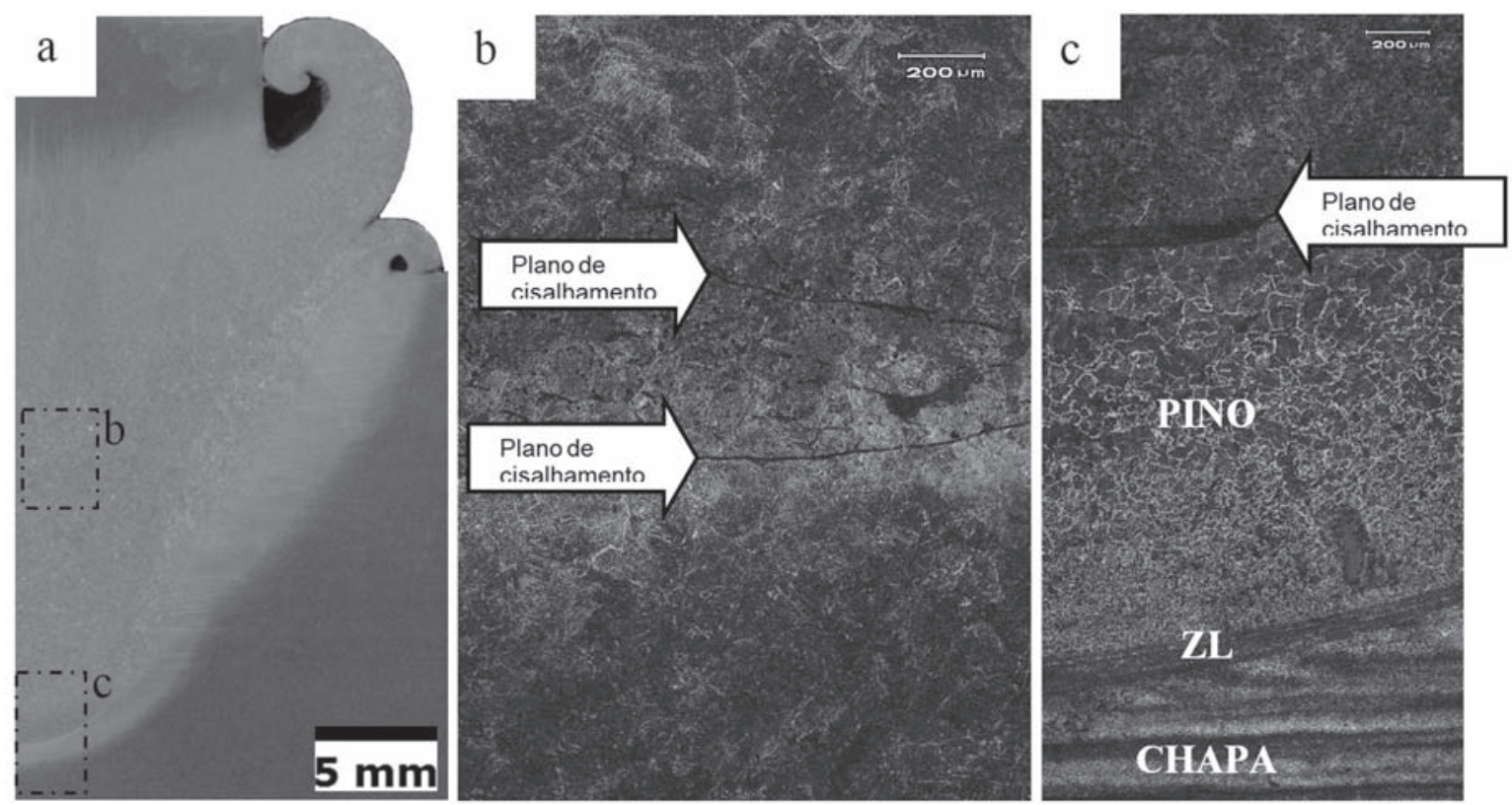

Figura 6 - Micrografias em ML da amostra de 50 kN: (a) macrografia que localiza as micrografias "b" e "c"; (b) planos de cisalhamento (setas 1,2 e 3); (c) micrografia mostrando a variação do TG austenítico a partir da ZL e plano de cisalhamento (seta). 

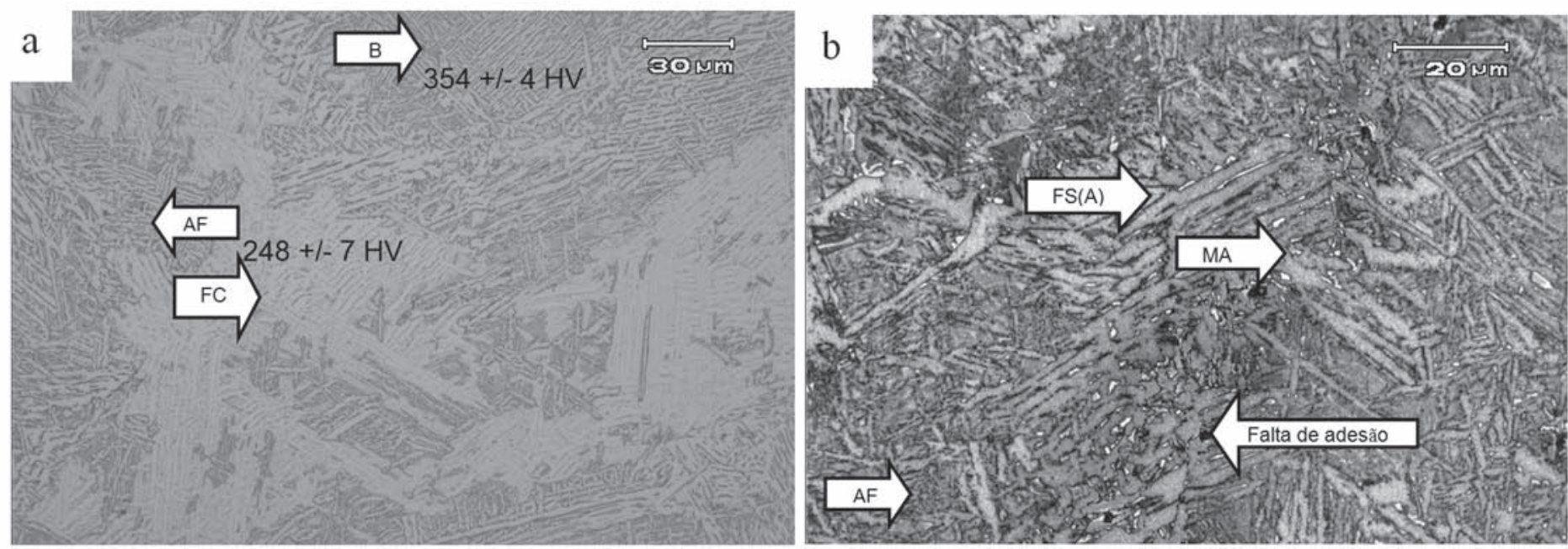

Figura 7 - Micrografia em ML. (a) ZL na carga de $50 \mathrm{kN}$ (500x). (b) Detalhe da ZL $50 \mathrm{kN}$ mostrando o constituinte MA, falta de adesão, Bainita [B], agregados de ferrita e carbonetos (perlita degenerada) [FC], ferrita acicular [AF], ferrita de Widmanstätten com segunda fase alinhada [FS(A)]. (1000x).

A micrografia da Figura 7 (a) foi feita na ZL à $10 \mathrm{~mm}$ da superfície da chapa. Ela mostra a heterogeneidade devido às diferentes microestruturas e microdurezas. Em destaque a Bainita com dureza de 354 +/- 4 HV, ferrita de Widmanstätten, "agregados de ferrita e carboneto" com 248 +/- 7 HV (uma definição de perlita e ferrita com carbonetos na interface - IIW International Institute of Welding [13]). Nota-se também ferrita acicular em menor proporção.

O constituinte MA (seta 1 - fase branca na Figura 7 b) é encontrada entre as placas das microestruturas e principalmente nas proximidades da ZL. Com o aumento da carga, o teor de constituinte MA diminui. Quantitativamente, foi obtido: $50 \mathrm{kN}$ - 0,375\% MA, $200 \mathrm{kN}-0,0759 \%$ MA e na carga $400 \mathrm{kN}$ o MA praticamente não foi observado. Segundo Ferry a formação de MA é facilitada pela maior quantidade de ferrita alotriomorfa presente na microestrutura; como a solubilidade do carbono na ferrita é muito baixa, ocorre enriquecimento da austenita adjacente com este elemento e com posterior resfriamento há formação de MA [15].

\subsection{Análise Microestrutural - 200 kN}

A concepção mais aceita da soldagem por atrito baseiase no fato que durante o contato das superfícies metálicas, as microprojeções de um corpo penetram nas cavidades do outro. Então forças maiores implicam em melhor adesão. Dependendo da carga aplicada tem-se a ocorrência de um escoamento intenso sob condições simultâneas de compressão e deslocamento de massa, que intensifica a ativação das superfícies em atrito [10]. Como pode ser visto na Figura 8, o ancoramento das microprojeções possibilita uma melhor adesão em relação a carga de $50 \mathrm{kN}$.
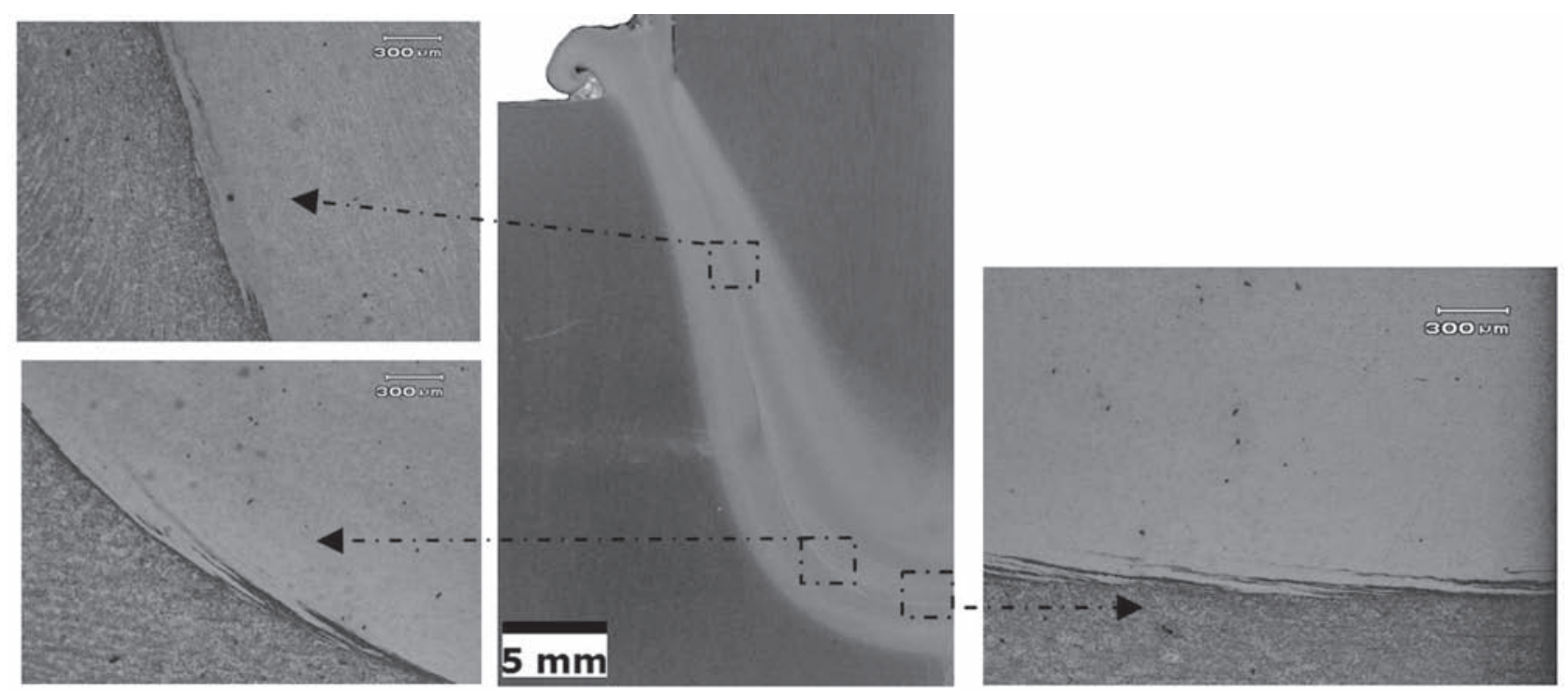

Figura 8 - Macrografia da junta soldada com carga $200 \mathrm{kN}$ no centro da figura e suas respectivas micrografias em ML mostrando as micro projeções de um corpo que penetraram nas cavidades do outro. 


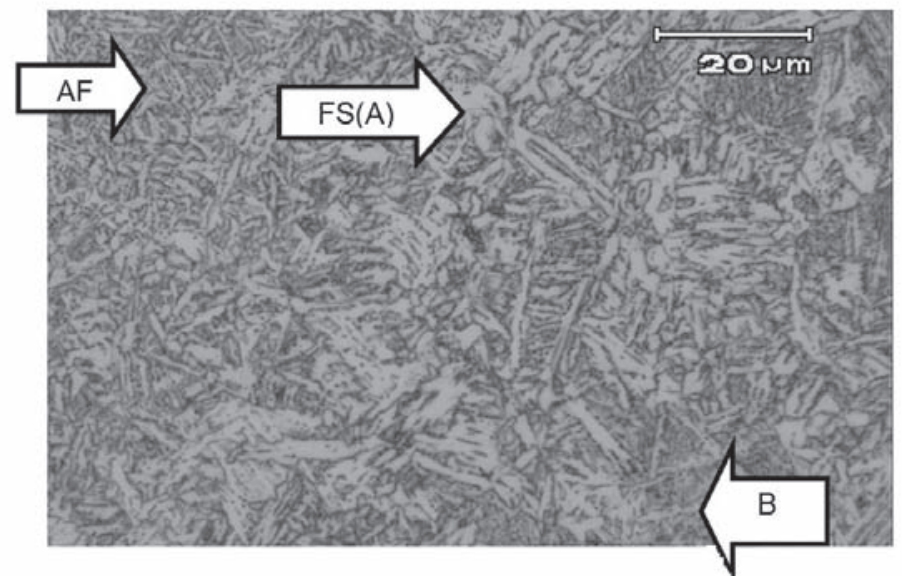

Figura 9 - Micrografia em ML da ZL na carga de 200 kN. ferrita acicular, ferrita de Widmanstätten, Bainita.
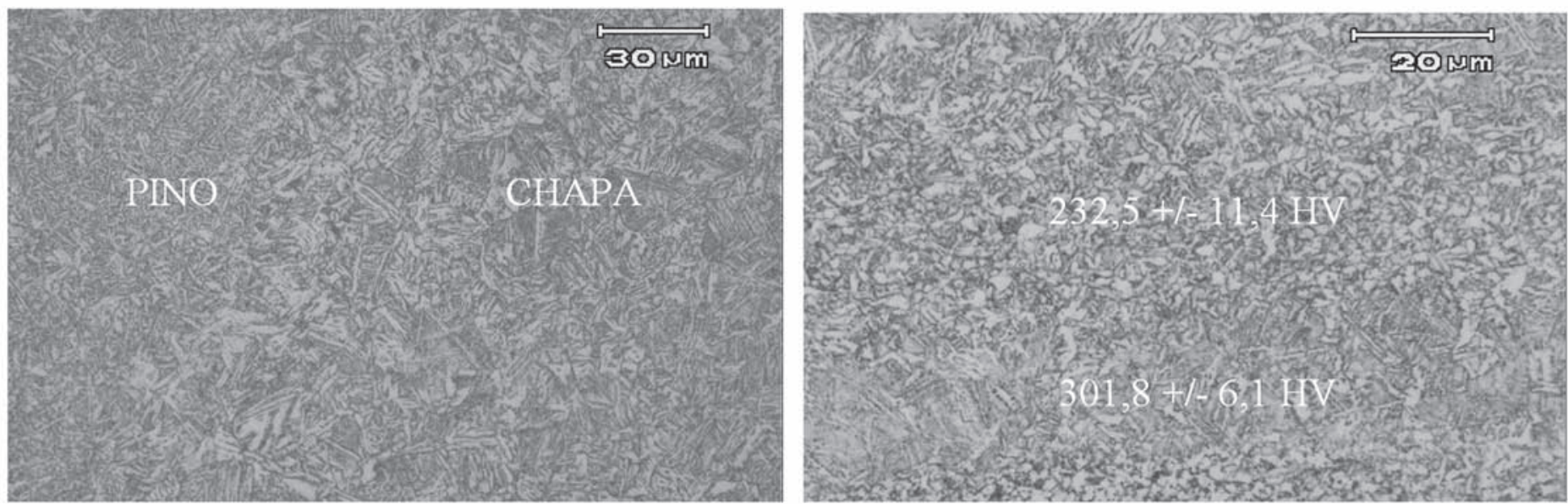

Figura 10 - Micrografica em ML. (a) ZL da amostra de carga 200 kN. (b) Microestrutura da chapa próxima ao pino do bandeamento transformado e suas diferentes microdurezas.

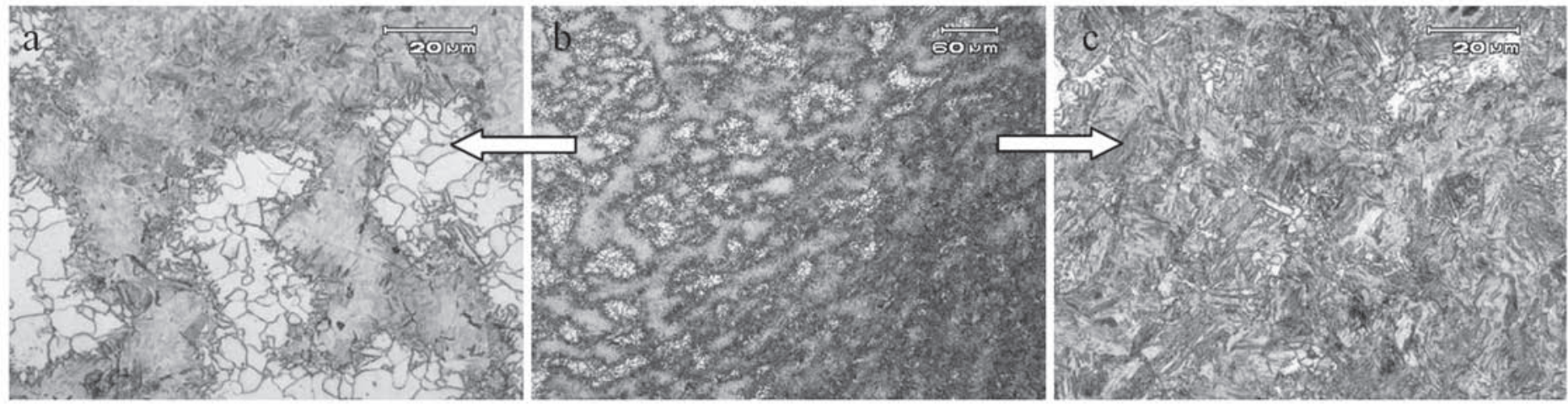

Figura 11 - Micrografias em ML. Em (a) região do pino transformada em bandas de martensita e ferrita (fase clara) na ZTMA (bandeamento horizontal-curvado), em (b) a mesma região porém com menor aumento e em (c) detalhe da martensita na ZTA (fase mais escura).

Evidenciou-se a formação de microestrutura heterogênea (Figura 9), com maior quantidade de Widmanstätten. O teor de ferrita acicular aumentou, mas insuficiente para produzir benefícios (Tabela 3). O lado do pino na ZL de carga $200 \mathrm{kN}$ uma microestrutura mais refinada que a da chapa (Figura 10 a). Foi observada a transformação do bandeamento da chapa próxima a ZL, pois onde seria a perlita, formou martensita (Figura 10 b). Nessa condição, ocorreu o bandeamento de ferritas muito refinadas e martensita com dureza 301,8 HV devido ao baixo percentual de carbono. As microestruturas aparecem mais refinadas em comparação com a carga axial de $50 \mathrm{kN}$.

\subsection{Análise Microestrutural - 400 kN}

Com a carga de $400 \mathrm{kN}$ foi possível reduzir a heterogeneidade das microestruturas formadas e praticamente extinguir o 

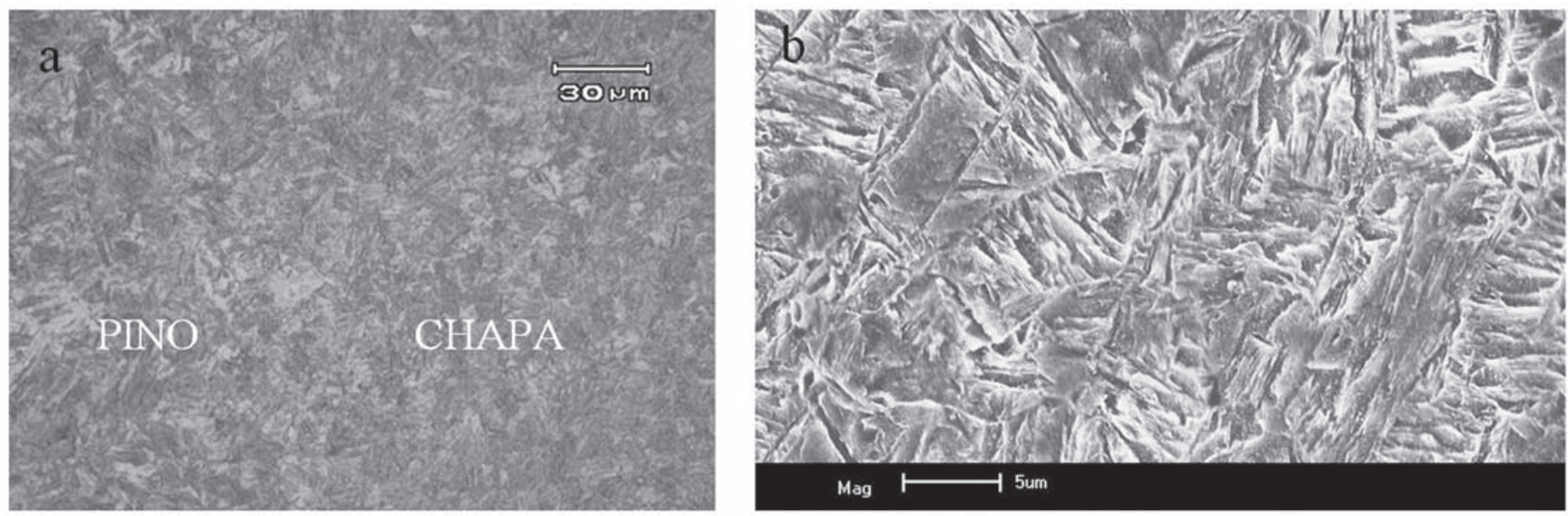

Figura 12 - (a) Micrografia em ML da ZL da junta soldada com carga $400 \mathrm{kN}$ e em (b) micrografia em MEV.

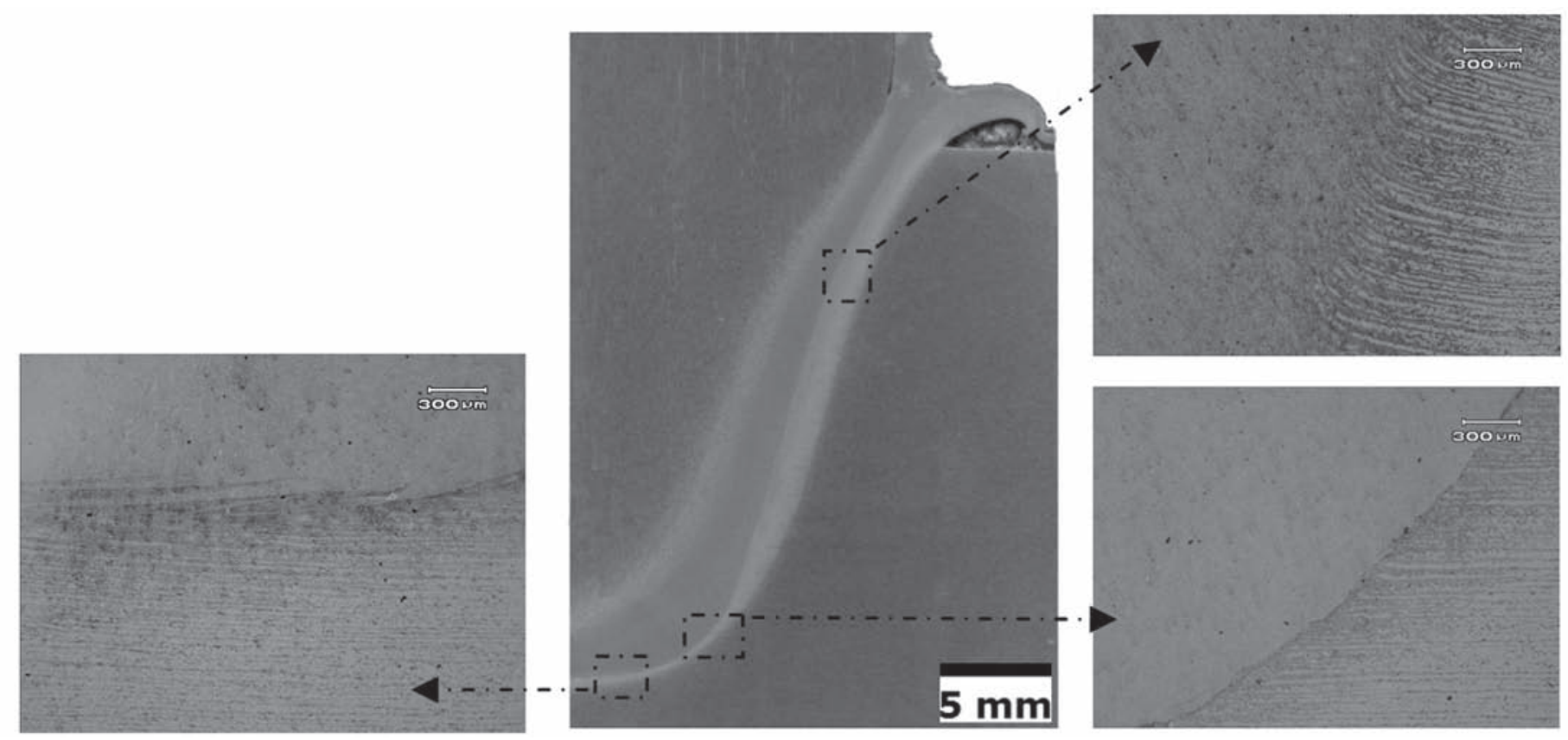

Figura 13 - Macrografia da junta soldada com carga $400 \mathrm{kN}$ no centro da figura e suas respectivas micrografias em ML mostrando as micro projeções de um corpo que penetraram nas cavidades do outro.

constituinte MA. Como pode ser visto na Tabela 3 houve a modificação da microestrutura que passou de grande quantidade de fases heterogêneas para uma microestrutura predominantemente martensítica. Juntamente com a martensita existe uma pequena fração de ferrita primária (Figura 12). A transformação do bandeamento ferrita-martensita é mais perceptível que a carga de $200 \mathrm{kN}$ (Figura 11). A banda que anteriormente era perlita transformou-se em martensita e a banda de ferrita permaneceu como tal. A junta soldada com carga de 400 kN, caracterizou-se como a melhor adesão (Figura 13) conforme previsto por LEBEDEV [11].

\subsection{Microdureza}

Os perfis de microdureza partem do MB (MB se origina na distância "0" nos gráficos) em direção ao pino, passando pelas zonas entre existentes entre esses. Abaixo os gráficos com os perfis de microdureza com setas destacando a ZL de cada condição (Figura 14). 

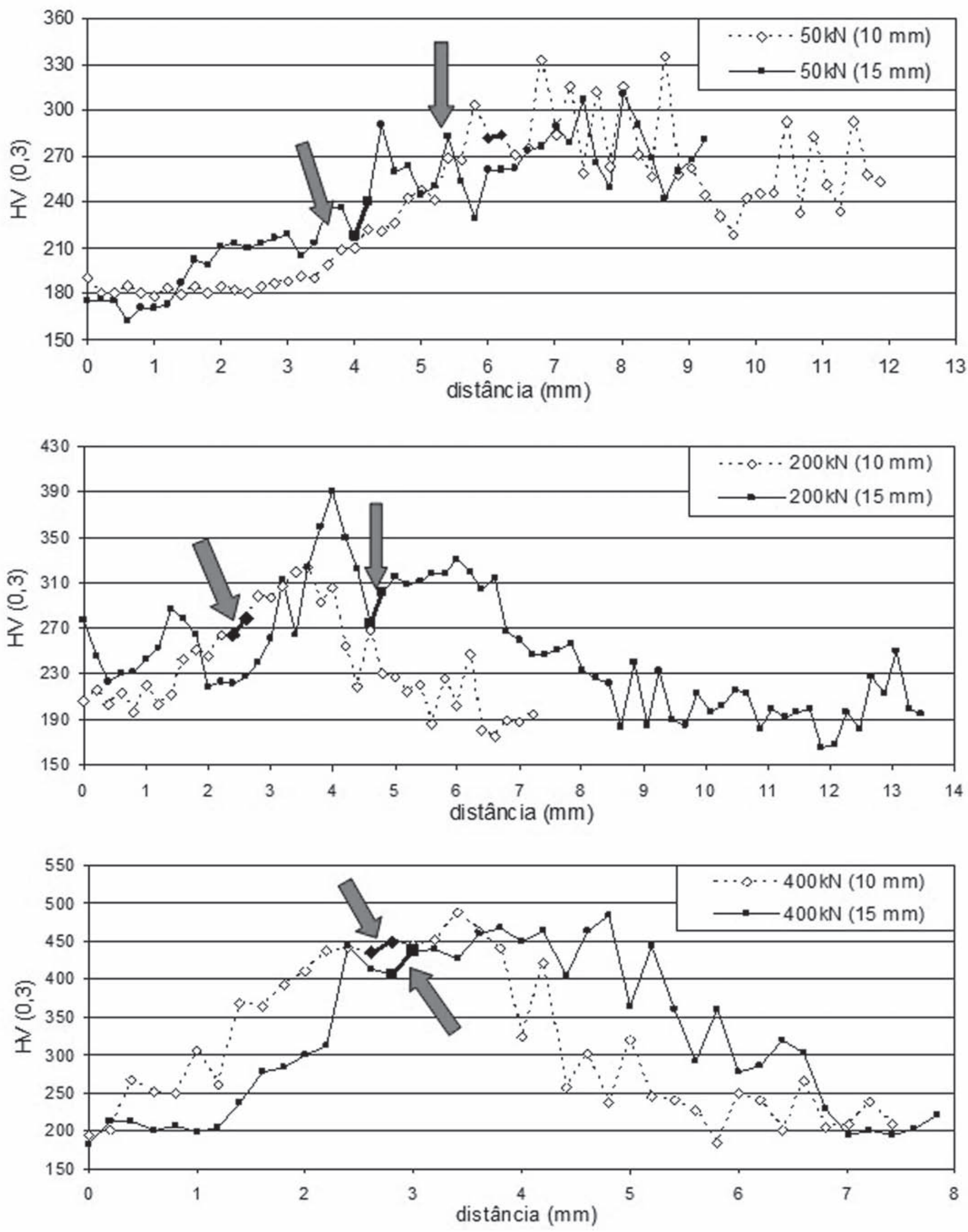

Figura 14 - Gráficos dos perfis de microdureza em relação à distância entre o MB/chapa (esquerda do gráfico) e o PINO (direita do gráfico) nas condições de $50 \mathrm{kN}, 200 \mathrm{kN}$ e $400 \mathrm{kN}$ para medidas em $10 \mathrm{~mm}$ e $15 \mathrm{~mm}$ a partir da superfície da chapa. 
Tabela 4 - Aspectos importantes dos gráficos de perfil de microdureza HV (0,3) a $10 \mathrm{~mm}$ da superfície. ZTA-MB (ZTA do Metal Base), ZTMA-MB (ZTMA do Metal Base), ZTMA-PINO (ZTMA do PINO), ZTA-PINO (ZTA do PINO).

\begin{tabular}{|c|cc|ccccc|}
\hline \multicolumn{2}{c}{$\mathbf{5 0 k N}$} & \multicolumn{2}{c}{$\mathbf{2 0 0 k N}$} & HV & $\Delta$ HV & HV & $\Delta$ 400kN \\
\hline HV & $\Delta$ HV & 125 & $204-214$ & 10 & $252-268$ & 16 \\
\hline ZT A-MB & $178-303$ & - & $167-295$ & 128 & $248-441$ & 193 \\
ZT MA-MB & - & - & $271-298$ & 27 & $444-450$ & 6 \\
Zona Ligação (ZL) & $271-333$ & 62 & $219-324$ & 105 & $257-487$ & 230 \\
ZT MA-PINO & - & - & $187-228$ & 41 & $244-319$ & 75 \\
ZT A-PINO & $219-335$ & 116 & & &
\end{tabular}

Tabela 5 - Aspectos importantes dos gráficos de perfil de microdureza HV $(0,3)$ a 15 mm da superfície.

\begin{tabular}{|c|c|c|c|c|c|c|}
\hline & \multicolumn{2}{|c|}{$50 \mathrm{kN}$} & \multicolumn{2}{|c|}{$200 \mathrm{kN}$} & \multicolumn{2}{|c|}{$400 \mathrm{kN}$} \\
\hline & HV & $\Delta \mathrm{HV}$ & HV & $\Delta \mathrm{HV}$ & HV & $\Delta \mathrm{HV}$ \\
\hline ZT A-MB & $162-237$ & 75 & $220-391$ & 171 & $199-212$ & 13 \\
\hline ZTMA-MB & - & - & - & - & $236-442$ & 206 \\
\hline Zona Ligação (ZL) & $218-240$ & 22 & 274-302 & 28 & $406-437$ & 31 \\
\hline ZTMA-PINO & - & - & - & - & $277-484$ & 207 \\
\hline ZT A-PINO & 229-311 & 82 & $166-331$ & 165 & $194-302$ & 108 \\
\hline
\end{tabular}

Tendo em vista o alto teor de Mn, que aumenta a temperabilidade do aço, se tornou possível a obtenção da microestrutura martensítica na ZTA-MB, no bandeamento transformado mencionado anteriormente, com microdureza máxima 391 HV na carga de 200 kN e 487 HV na carga de 400 $\mathrm{kN}$. Provavelmente foi atingida uma maior dureza na maior carga devido à maior taxa de resfriamento que possibilita a formação de maior quantidade de martensítica. O MB por ser constituído de bandeamentos de perlita e ferrita, tem menor microdureza. À medida que o perfil avança, aumenta a microdureza devido o surgimento diferentes fases de acordo com cada condição anteriormente avaliada, que volta a cair quando se aproxima do pino que também é constituído por bandeamento de ferrita e perlita [1].

\section{Conclusões}

Os resultados obtidos ao longo do estudo da microestrutura formada no processo de soldagem por atrito em aço com pino consumível com as cargas de 50, 200 e $400 \mathrm{kN}$ levaram as seguintes conclusões:

- Forças maiores contribuem para obtenção de uma microestrutura mais refinada, evitam a formação heterogênea de fases (tais como Widmanstätten, bainita e agregados de carbonetos) e possibilitam a formação predominantemente martensítica, que apesar de ser frágil, é menos deletéria que as microestruturas anteriores.

- Maiores cargas resultaram numa maior taxa de resfriamento, num menor tempo de processamento, numa menor área da ZTA e num menor teor de constituinte MA. O tempo de processamento da carga de $50 \mathrm{kN}$ é muito maior em relação às outras cargas;

- Na carga de $50 \mathrm{kN}$ houve falta de adesão ao longo da ZL e o fenômeno de cisalhamento a quente do pino, ambos defeitos que degradam as propriedades da tentativa em obter uma junta soldada, enquanto que nas cargas de $200 \mathrm{kN}$ e $400 \mathrm{kN}$ isso não ocorreu;
- Nas amostras de carga $200 \mathrm{kN}$ e $400 \mathrm{kN}$ obteve-se um bandeamento de martensita e ferrita, com melhor percepção das bandas na carga de $400 \mathrm{kN}$;

- O perfil de microdureza aumenta o valor absoluto quando cruza pela ZL do MB para o pino em todas as condições. E os valores de microdureza dos perfis apresentaram boa correlação com as respectivas microestruturas encontradas.

\section{Referências Bibliográficas}

[1] Unfried, J; Hermenegildo, T. F; PAES, M. T. P; Pope, A. M; Ramirez, A. J; "Influence of Process Parameters in the TMAZ Microstructural Evolution of C-Mn Steels Friction Hydro-Pillar Welded Joints". Proceedings of the 8th International Conference on Trends in Welding Research. Pine Mountain, Geórgia USA. June 2008.

[2] BLAKEMORE, G. R. Applications of state of the art portable friction welding equipment euro-join. In: EUROPEAN CONFERENCE ON JOINING TECHNOLOGY, 2., 1984, Florence; Italy. Genova: Istituto Italiano della Saldatura, 1994. p. 127-36.

[3] NICHOLAS, E. D. "Friction processing technologies". Welding in the World, v. 47, n. 11-12, p. 2-9, Nov./Dec. 2003.

[4] BLAKEMORE, G. R. Friction welding-technology for the new millennium. In: OFFSHORE TECHNOLOGY CONFERENCE, 31., 1999, Houston, Texas. [S.n.t.]. Paper 11063-MS

[5] ANDREWS, R. E.; MITCHELL, J. S. Underwater repair by friction stitch welding. Metals and Materials, v. 6, n. 12, p. 7967, Dec. 1990.

[6] MEYER, A. Friction Hydro Pillar Processing. Dr.-Ing. Thesis an der Technischen Universität Braunschweig, Hamburg, 2002.

[7] Pinheiro, G. A. "Local Reinforcement of Magnesium Components by Friction Processing: Determination of Bonding Mechanisms and Assessment of Joint Properties". GKSS, Forschungszentrum Geesthacht GmbH, Geesthacht, 2008.

[8] NICHOLAS, E.D. FRICTION PROCESSING TECHNOLOGIES - Friction and Forge Processes Group, TWI 
Ltd. , UK, 2003.

[9] PAES, M. T. P; Características fundamentais de soldas por atrito tipo FHPP. Exame de qualificação para doutorado. UFRJ, 2009, 56 páginas.

[10] KRAUSS, G. "Solidification, segregation, and banding in carbon and alloy steels". Journal Metallurgical and Materials Transactions B. Vol. 34, Number 6, December, 2003

[11] LEBEDEV, V. K.; CHERNENKO, I. A. Friction Welding. Sov. Tech. Ver. 1992. v. 4. p. $59-168$.

[12] ELLIS C. R. G., Continuous Drive Friction Welding of Mil Steel, Welding Journal, p 183s- 197s, Apr. 1972.

[13] Committee of Welding Metallurgy of Japan Welding Society. "Classification of Microstructures in Low C - Low Alloy Steel Weld Metal and Terminology“. IIW Doc. IX - 128283. 22p.

[14] G. Thewli., "Classification and quantification of microstructures in steels". Journal Materials Science and Technology. Vol. 20, pages 143-160, February, 2004.

[15] Ferry, M., Thompson, M. e Manohar, P.A. Decomposition of coarse grained austenite during accelerated cooling of C-Mn steels. ISIJ International. 2002, Vol. 42, n. 1, 86-93. 